\title{
Anti-CD11b antibody treatment suppresses the osteoclast generation, inflammatory cell infiltration, and autoantibody production in arthritis-prone FcyRIIB-deficient mice
}

Mareki Ohtsuji ${ }^{1}$, Qingshun Lin ${ }^{1}$, Hideki Okazaki ${ }^{2}$, Kazuko Takahashi ${ }^{3}$, Hirofumi Amano ${ }^{4}$, Hideo Yagita ${ }^{5}$, Hiroyuki Nishimura ${ }^{1}$ and Sachiko Hirose ${ }^{1 *}$

\begin{abstract}
Background: Previously we established an arthritis-prone FcyRIIB-deficient mouse strain (designated KO1). Anti-mouse CD11b mAb (5C6) has been reported to inhibit the recruitment of peripheral CD11 b ${ }^{+}$myelomonocytic cells from the blood to the inflammatory site. These cells include neutrophils and monocytes, both of which play important roles in the development of arthritis. Here we treated $\mathrm{KO} 1$ mice with $5 \mathrm{C} 6 \mathrm{mAb}$ in order to study its effect on arthritis development.
\end{abstract}

Methods: To evaluate the disease-preventive effect of 5C6, 4-month-old preclinical KO1 mice were divided into three groups: the first treated with 5C6 for 6 months, the second treated with normal rat lgG for 6 months, as a control, and the third left untreated. Arthritis severity and immunological abnormalities were compared among the groups, along with transcriptional levels of several important arthritis-related factors in ankle joints, spleen, and peripheral blood cells.

Results: The $5 \mathrm{C} 6$ treatment ameliorated arthritis in $\mathrm{KO} 1$ mice, showing decreases in inflammatory cell infiltration and osteoclast formation. Analysis of transcriptional levels in ankle joints revealed that compared with the two control groups, the 5C6-treated group showed downregulated expression of RANK, RANKL, MCP-1, RANTES, TNFa, and IL-6, and at the same time showed significantly up-regulated expression of the decoy receptor for RANKL, i.e. osteoprotegerin. In addition, the disease suppression was associated with the lower serum levels of autoantibodies, and the decreased frequencies of activated B cells and plasma cells. The expression levels of B cell activation/ differentiation-related cytokines were suppressed in spleen and peripheral leukocytes of the 5C6-treated mice. Intriguingly, while untreated KO1 mice spontaneously developed marked monocytosis, the 5C6-treated mice showed the significantly down-regulated frequency of monocytes.

Conclusions: The outcome of 5C6 treatment was complex, in which the 5C6-mediated disease-preventive effect is likely due on one hand to the decrease in the recruitment of inflammatory cells and osteoclast precursor monocytes from the periphery into the joints, and on the other hand to the suppression of B cell activation/maturation and of autoantibody production via the suppression of B cell stimulating cytokine production. The lower levels of these cytokines may be the secondary effect of the lower frequency of monocytes, since monocytes/macrophages are the major producers of these cytokines.

Keywords: Rheumatoid arthritis, FcyRIIB deficiency, Osteoclast, Monocytes, B cell activation

\footnotetext{
* Correspondence: sacchi@toin.ac.jp

${ }^{1}$ Department of Biomedical Engineering, Toin University of Yokohama, 1614

Kurogane-cho, Aoba-ku, Yokohama 225-8502, Japan

Full list of author information is available at the end of the article
} 


\section{Background}

Rheumatoid arthritis (RA) is a chronic systemic autoimmune disease, characterized by synovitis with synovial lining cell hyperplasia and marked inflammatory cell infiltration in multiple joints in the initial stage; followed by marked pannus formation and progressive destruction of cartilage and bone mediated by augmented proliferation of activated osteoclasts. Osteoclasts are multinucleated giant cells positive for tartrate-resistant acid phosphatase (TRAP) and cathepsin $K$, and they resorb bone matrix $[1,2]$. These cells differentiate from the osteoclast precursors of monocyte/macrophage lineage cells in the bone marrow [3] and in peripheral blood [4]. The process of osteoclastogenesis is controlled by the interaction of receptor activator of $\mathrm{NF}-\mathrm{kB}$ (RANK) expressed on osteoclast precursors with its ligand RANKL expressed on synovial fibroblasts, osteoblasts, and T helper 17 (Th17) cells [5-7]. The RANKLmediated osteoclastogenesis is counterbalanced by the physiologically expressed decoy receptor, osteoprotegerin (OPG) [8]. Accumulating evidence has indicated that high expression levels of proinflammatory cytokines, such as tumor necrosis factor $\alpha$ (TNF $\alpha)$, interleukin (IL)-1, IL-6, and IL-17, in inflamed synovial tissues may play pivotal roles in the processes of both synovitis and osteoclastogenesis [9].

We previously found that an inhibitory IgG Fc receptor IIB (FcyRIIB)-deficient mouse strain, originally established on $129 \times$ C57BL/6 (B6) genetic background and backcrossed to $\mathrm{B} 6$ mice, designated $\mathrm{KO} 1$, spontaneously develops human RA-like disease features with marked synovitis and severe cartilage/bone destruction in multiple joints [10]. Signal blockade of IL-6 or TNF $\alpha$ markedly ameliorates arthritis in KO1 mice [11, 12]. Also, intriguingly, the severity of arthritis was positively associated with the increased frequency of peripheral monocytes in KO1 mice [11]. Since peripheral monocytes are composed of several subsets, including inflammatory cells and osteoclast precursors [4], the high frequency of peripheral monocytes may contribute to the up-regulation of both inflammatory cell infiltration and osteoclastogenesis in the arthritic joints of KO1 mice.

The rat anti-mouse CD11b mAb, 5C6, has been shown

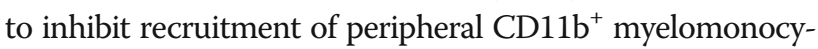
tic cells into the inflamed lesion [13]. These CD11b ${ }^{+}$myelomonocytic cells include neutrophils and monocytes, both of which play important roles in the initiation and progression of arthritis $[11,14]$. We treated KO1 mice with 5C6 to evaluate the arthritis-preventive potential of $5 \mathrm{C} 6 \mathrm{mAb}$ in $\mathrm{KO} 1$ mice. The 5C6 treatment ameliorated arthritis in $\mathrm{KO} 1$ mice with decrease in inflammatory cell infiltrations, osteoclast formation, and RA-related cytokine production in the joint tissues. The $5 \mathrm{C} 6$ treatment also suppressed B cell activation/differentiation and autoantibody production. The possible mechanism in which 5C6 treatment ameliorates disease severity is discussed.

\section{Methods \\ Mice}

Arthritis-prone KO1 is a FcyRIIB-deficient B6 congenic line [10], obtained by backcrossing the originally constructed Fc $\gamma$ RIIB-deficient mice on a hybrid $(129 \times$ B6) background into a B6 background for over 12 generations. C57BL/6 (B6) mice were purchased from Japan SLC, Inc., and used as the arthritis-free normal control. Only female mice were analyzed in the present study. All mice were housed under identical specific-pathogenfree conditions. All animal experiments were performed in accordance with the animal experiment guidelines of our university.

\section{Incidence and scoring of arthritis}

Ankle joint swelling was examined by eye inspection twice a month after 5 months of age and arbitrarily scored as follows: 0 , no swelling; 1 , mild swelling; 2 , moderate swelling; and 3, severe swelling. Scores for both ankle joints were summed for each mouse, and mice with a score of 2 or over were considered positive for arthritis. In addition to eye inspection, the degree of swelling in the region of the tarsal bones was measured at 10 months of age under anesthesia using a Vernier micrometer.

\section{In vivo administration of anti-CD11b mAb (5C6)}

To examine the preventive effect of mAb $5 \mathrm{C} 6$ on the development of arthritis, 4-month-old preclinical KO1 mice were randomly divided into three groups. Each group of 15 mice was left untreated, treated with normal rat IgG (Sigma Chemical Co.), or treated with rat antimouse CD11b mAb (5C6, rat IgG2b [13]). Two hundred micrograms of rat IgG or 5C6 was administrated intraperitoneal (i.p.) twice a week for 6 months.

\section{Histopathology}

Joint tissues were decalcified in 10\% EDTA in $0.1 \mathrm{M}$ Tris buffer ( $\mathrm{pH} 7.4$ ), fixed in 4\% paraformaldehyde, and embedded in paraffin. Tissue sections were stained with hematoxylin/eosin, and also stained for TRAP using the TRAP/ALP stain kit (Wako Pure Chemical Industries Ltd.).

\section{Serum levels of antibodies}

Serum levels of IgG anti-cyclic citrullinated peptide (CCP) antibodies were measured employing commercially available kits (Cosmic Corporation) using antimouse IgG second antibodies and were expressed as relative units according to the manufacturer's instructions. Serum levels of rheumatoid factor (RF) were measured using an ELISA, as previously described [15]. Briefly, an ELISA plate pre-coated with mouse IgG Fc 
fragment (OEM Concepts) was incubated with appropriately diluted mouse serum samples, washed, and then incubated with peroxidase-conjugated rat anti-mouse $\mathrm{k}$ chain antibodies (BD Biosciences Pharmingen). RF activity was expressed in units referring to a standard curve obtained by serial dilution of a standard serum pool from 4-6-month-old MRL/lpr mice containing 1000 unit activities/ml. Serum IgG anti-double-stranded (ds) DNA was measured using an ELISA plate pre-coated with $5 \mu \mathrm{g} / \mathrm{ml}$ calf thymus dsDNA (Sigma-Aldrich). DNA-binding activity was expressed in units as previously described [10].

\section{Flow cytometric analysis}

Spleen cells were stained with phycoerythrin (PE)-labeled anti-B220 (RA3-6B2) mAb, allophycocyanin (APC)labeled anti-CD69 (H1.2 F3), anti-CD138 (281-2), and anti-CD11c (HL3) mAbs, fluorescein isothiocyanate (FITC)-labeled anti-CD4 (RM4-5) and anti-CD11b (M1/70) mAbs, and FITC-labeled peanut agglutinin (PNA). For peripheral monocyte staining, peripheral leukocytes were stained with FITC-labeled anti-CD11b, PE-labeled antiGr-1 (RB6-8C5), and APC-labeled CD115 (AFS98) mAbs. Fluorescent-labeled reagents were purchased from Bay Bioscience (B220, CD4, CD11b, Gr-1, CD115), Bio Legend (CD69, CD138), BD Bioscience (CD11c), and SigmaAldrich (PNA). Stained cells were analyzed using a FACSAria cytometer and FlowJo software (Tree Star Inc.). Auto-fluorescence was considered as negative control.
Compensation of spillover was performed according to the manufacturer's instructiongei.

\section{Quantitative real-time PCR (qRT-PCR) analysis}

Total RNA was isolated from ankle joint tissue of the tarsal bones containing soft tissue and bone/cartilage/marrow, from the spleen, and from peripheral leukocytes using QIAGEN RNeasy Lipid Tissue Minikit (Cat. number 74804). Briefly, $25 \mathrm{mg}$ of ankle joint tissue, spleen, or leukocyte pellet was added in $500 \mu \mathrm{l}$ of QIAzol lysis reagent in a 2-ml tube containing 5-mm-diameter zirconia beads (Hirasawa YTZ-5) and homogenized on TissueLyser (Qiagen) for 1 min at $30 \mathrm{~Hz}$. Total RNA was extracted from homogenized materials using Minikit according to the manufacturer's instructions, and $0.5 \mu \mathrm{g}$ of total RNA was used to synthesize the single-stranded cDNA using an oligo (dT)-primer with Superscript III First-Strand Synthesis kit (Invitrogen). The cDNA product was used for qRT-PCR. The data were normalized to $\beta$-actin as a reference. The primer pairs used and the length of PCR products are shown in Table 1. The quantity was normalized using $2^{-\Delta \Delta C T}$ methods.

\section{Statistics}

The Kaplan-Meier method was used to evaluate differences in the incidence of arthritis. Analysis of variance (ANOVA) was used to make comparisons among three or four groups of mice, and Student's $t$ test was used to compare two groups of mice. A value of $P<0.05$ was considered as statistically significant.

Table 1 Primer pairs used in qRT-PCR and size of PCR products

\begin{tabular}{|c|c|c|c|}
\hline Gene for & $\begin{array}{l}\text { Forward } \\
\left(5^{\prime} \text { to } 3^{\prime}\right)\end{array}$ & $\begin{array}{l}\text { Reverse } \\
\left(5^{\prime} \text { to } 3^{\prime}\right)\end{array}$ & $\begin{array}{l}\text { Size } \\
\text { (bp) }\end{array}$ \\
\hline RANK & GCTGGCTACCACTGGAACTC & GTGCAGTTGGTCCAAGGTTT & 182 \\
\hline RANKL & TGTACTTTCGAGCGCAGATG & AGGCTTGTTTCATCCTCCTG & 160 \\
\hline OPG & TGAGTGTGAGGAAGGGCGTTA & CCATCTGGACATIITITGCAAA & 130 \\
\hline MCP-1 & AGGTCCCTGTCATGCTTCTG & TCTGGACCCATTCCTTCTTG & 249 \\
\hline CX3CL1 & CGCGTTCTTCCATTTGTGTA & CTGTGTCGTCTCCAGGACAA & 169 \\
\hline RANTES & TCGTGCCCACGTCAAGGAGTATT & TCTTCTCTGGGTTGGCACACACTT & 107 \\
\hline TNFa & TATGGCCCAGACCCTCAC & GGTTGTCTTTGAGATCCATGC & 110 \\
\hline IL-6 & GAGGATACCACTCCCAACAGACC & AAGTGCATCATCGTTGTTCATACA & 141 \\
\hline |L-17 & TCTCTGATGCTGTTGCTGCT & GACCAGGATCTCTTGCTGGA & 346 \\
\hline IL-10 & CAGCCGGGAAGACAATAACT & GTTGTCCAGCTGGTCCTTTG & 121 \\
\hline BAFF & TTGTCCAGCAGTTCACAGC & CCGGTGTCAGGAGTTTGACT & 160 \\
\hline IL-1 $\beta$ & GCCCATCCTCTGTGACTCAT & AGGCCACAGGTATTTTGTCG & 230 \\
\hline BSF-3 & CGAGCCTGACTTCAATCCTC & TACGTCGGAGTTCAGCTGTG & 185 \\
\hline IFNY & AAGACAATCAGGCCATCAGC & ATCAGCAGCGACTCCTTTTC & 225 \\
\hline$\beta$-actin & TGGGTATGGAATCCTGTGG & GTACTTGCGCTCAGGAGGAG & 200 \\
\hline
\end{tabular}

RANK receptor activator of NF-KB, RANKL RANK ligand, OPG osteoprotogerin, $M C P-1$ monocyte chemotactic protein-1, RANTES regulated on activation, normal T cell expressed and secreted, BAFF B-cell-activating factor of the tumor-necrosis-factor family, BSF-3 B cell stimulating factor, IFN- $\gamma$ interferon- $\gamma, b p$ base pairs 


\section{Results}

\section{Suppressive effect of $5 \mathrm{C} 6$ treatment on severity of} arthritis

Four-month-old $\mathrm{KO} 1$ mice in the preclinical stage were divided into three groups, namely untreated, normal rat IgG-treated, and 5C6-treated groups. Figure 1a compares the cumulative incidence of arthritis and the ageassociated changes in the arthritis score. Untreated KO1 mice spontaneously developed arthritis with bilateral swelling of the ankle joints after 5 months of age, and the severity of arthritis increased with age. KO1 mice treated with normal rat IgG developed severe arthritis similar to those observed in untreated $\mathrm{KO} 1$ mice. In contrast, in 5C6-treated $\mathrm{KO} 1$ mice the incidence and severity of arthritis were markedly suppressed from the initiation of arthritic inflammation. Although the incidence and severity were marginally higher in 5C6treated $\mathrm{KO} 1$ mice compared with those in normal B6 mice, no significant difference was observed. Figure $1 b$ shows the measurement of ankle joint width in the region of the tarsal bones at 10 months of age. The increased width due to joint inflammation observed in untreated and normal rat IgG-treated mice was significantly suppressed in 5C6-treated KO1 mice. Figure 1c shows representative macroscopic and histological findings in the hind paws in the three groups of mice at 10

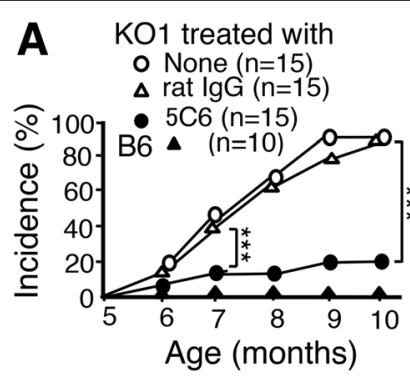

C
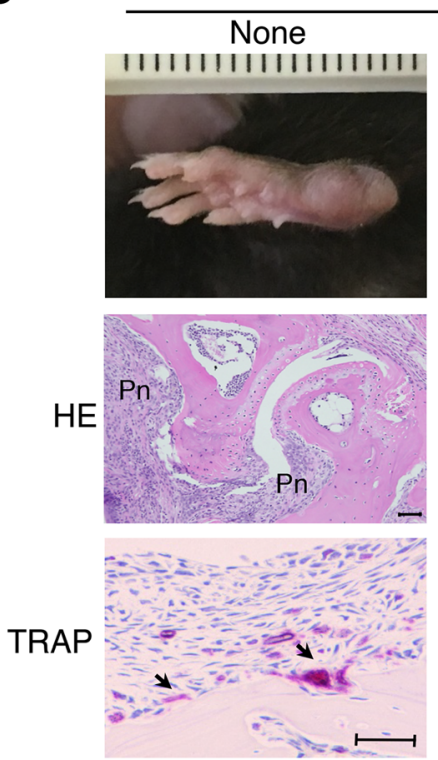

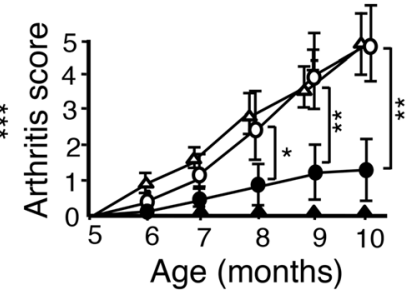

KO1 treated with
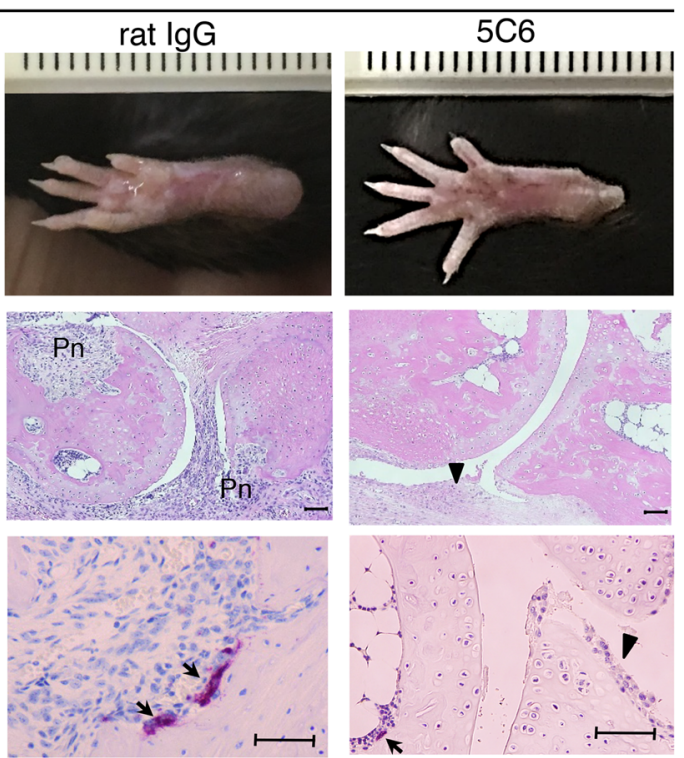

Fig. 1 Preventive effect of anti-mouse CD11b monoclonal antibody (5C6) treatment. a Comparisons of the cumulative incidence of arthritis and the age-associated changes in arthritis score (mean and SE) among untreated $(n=15)$, normal rat IgG-treated $(n=15), 5 C 6$-treated FcyRllB-deficient mouse strain $(K O 1)(n=15)$, and B6 mice $(n=10)$. The incidence was significantly lower in 5C6-treated mice, compared with those in untreated and normal rat lgG-treated mice after 7 months of age (Kaplan-Meier method ${ }^{* *} P<0.001$ ), and the arthritis score was significantly suppressed in 5C6-treated mice, compared with those in untreated and normal rat IgG-treated mice after 8 months of age (analysis of variance (ANOVA) ${ }^{*} P<0.05$, ${ }^{*} P<0.01$ ). b Comparisons of the width of the ankle joints at the tarsal bones (mean and SE) of at least 6 mice at 10 months of age among untreated, normal rat IgG-treated, 5C6-treated KO1, and B6 mice. Statistical significance is shown (ANOVA *** < 0.001). c Representative macroscopic and histopathological findings in the hind paws in untreated, normal rat lgG-treated, and 5C6-treated KO1 mice at 10 months of age. Untreated and normal rat lgG-treated KO1 mice had marked synovitis with inflammatory cell infiltration, pannus formation (Pn), tartrate-resistant acid phosphatase (TRAP)-positive osteoclast generation (arrowhead), and the destruction of cartilage and bone. The 5C6-treated KO1 mice only had mild synovial lining cell proliferation (triangle) and a few small TRAP-positive osteoclasts in bone marrow (arrowhead). Representative results obtained from six female mice in each group.

Hematoxylin/eosin (HE) and TRAP staining. Bars $=50 \mu \mathrm{m}$ 
months of age. Untreated and normal rat IgG-treated mice had marked swelling of the ankle joints, with severe synovitis and remarkable pannus formation, along with increases in the number of TRAP-positive osteoclasts at the resorption lacuna on the surface of cartilage and bone. The 5C6-treated KO1 mice developed no such distinctive changes and only had mild synovial lining cell proliferation. A few TRAP-positive small osteoclasts were observed in the bone marrow, but destructive changes of bone/cartilage were seldom observed.

\section{Effects of 5C6 treatment on serum autoantibody levels, lymphocyte activation status, and peripheral leukocyte frequencies}

Figure 2 compares the serum levels of RF, and IgG autoantibodies against CCP and dsDNA among three groups of mice at 8 months of age. Levels of all these antibodies were significantly suppressed in 5C6-treated $\mathrm{KO} 1$ mice, compared with those in untreated and normal rat IgG-treated KO1 mice.

We then examined the spleen weight, and the frequencies of spleen cell subpopulations and of peripheral leukocyte subsets in 10-month-old mice. As shown in Table 2, no differences were observed in the spleen weight among the three groups. Interestingly, in 5C6treated mice, while the total $\mathrm{B}$ cell frequency was significantly higher, the frequencies of $\mathrm{CD} 69^{+}$activated $\mathrm{B}$ cells, $\mathrm{PNA}^{+}$germinal center $\mathrm{B}$ cells, and $\mathrm{CD} 138^{+}$plasma cells were significantly lower, compared with those in the two control groups of mice. Figure 3a shows the representative flow cytometric profiles of spleen cells stained with CD69/B220, PNA/B220, and CD138/B220 in untreated and 5C6-treated $\mathrm{KO} 1$ mice. It is likely that in the control $\mathrm{KO} 1$ mice the accelerated $\mathrm{B}$ cell differentiation leads to the decrease in the frequencies of total $\mathrm{B}$ cells, and that this phenomenon is suppressed by 5C6 treatment. As for $\mathrm{T}$ cells, no significant differences were seen in the frequencies of total $\mathrm{T}$ cells or $\mathrm{CD} 9^{+}$activated $\mathrm{T}$ cells among the three groups of mice. The frequencies of $\mathrm{CD}_{11 \mathrm{~b}^{+}}$macrophages and $\mathrm{CD} 11 \mathrm{c}^{+}$dendritic cells were comparable between untreated and 5C6-treated groups of mice. We also examined the effect of 5C6 treatment on the frequencies of peripheral leukocyte subsets. As $5 \mathrm{C} 6 \mathrm{mAb}$ has been shown to inhibit the recruitment of peripheral $\mathrm{CD}_{11 \mathrm{~b}^{+}}$myelomonocytic cells into an inflamed lesion [13], it was suggested that the frequencies of peripheral $\mathrm{CD} 11 \mathrm{~b}^{+}$cells including neutrophils and monocytes might be increased in 5C6-treated $\mathrm{KO} 1$ mice. However, our present study showed that the frequency of neutrophils tended to be decreased in 5C6-treated $\mathrm{KO} 1$ mice, although there was no statistical significance. Moreover, the frequency of monocytes, especially $\mathrm{Gr}-1^{-}$ monocytes, was significantly decreased in 5C6-treated KO1 mice (Table 2). Thus, in addition to the inhibition of $\mathrm{CD}_{11 \mathrm{~b}^{+}}$myelomonocytic cell recruitment from vessels, 5C6 may have been somewhat cytotoxic in our experiment. The original report showed that $5 \mathrm{C} 6$ did not induce cell death [13]. This discrepancy may be due to our injection protocol, twice a week for 6 months, which is different to the single injection used in the original study. The representative flow cytometric profiles of peripheral leukocytes stained with $\mathrm{Gr}-1 / \mathrm{CD} 11 \mathrm{~b}$ are shown in Fig. 3b. Neutrophils had the highest Gr-1 expression level, while monocytes were divided into two populations, $\mathrm{Gr}-1^{+}$and $\mathrm{Gr}-1^{-}$subsets. These two monocyte subsets were clearly shown by staining peripheral leukocytes with CD115/Gr-1/CD11b and by examining $\mathrm{Gr}-1 / \mathrm{CD} 11 \mathrm{~b}$ expression profiles of $\mathrm{CD} 115^{+}$monocytes (Fig. 3c).

\section{qRT-PCR analysis for mRNA expression levels of RANK/} RANKL/OPG and arthritis-related cytokines in ankle joints To understand the mechanism of the suppression of arthritis in 5C6-treated KO1 mice, transcription levels of RANK, RANKL, OPG, and several important arthritisrelated cytokines in ankle joint tissues were compared among the three groups of mice by qRT-PCR analysis using mRNA extracted from tarsal bones of the mice at 10 months old. Data on normal B6 mice were used as a relative control, and the expression levels in the three
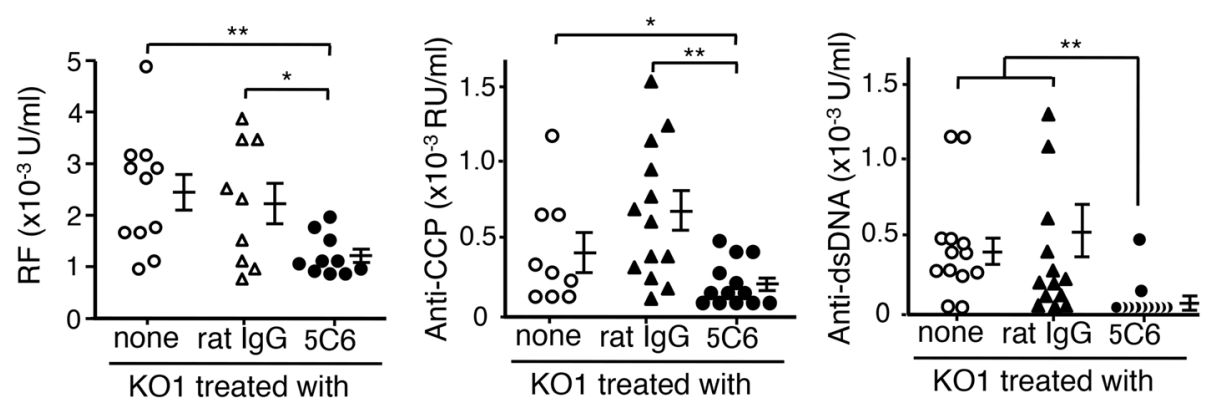

Fig. 2 Comparisons of serum levels of rheumatoid factor (RF), and IgG autoantibodies against citric citrullinated peptide (CCP) and dsDNA among untreated, normal rat IgG-treated, and anti-mouse CD11b monoclonal antibody (5C6)-treated FcyRIIB-deficient mouse strain (KO1) mice at 8 months of age. The mean and SE are shown with statistical significance (analysis of variance ${ }^{*} P<0.05,{ }^{* *} P<0.01$ ) 
Table 2 Spleen weight and frequencies of spleen cell subpopulations and peripheral leukocyte subsets in untreated, normal rat lgG-treated, and 5C6-treated KO1 mice ${ }^{a}$

\begin{tabular}{|c|c|c|c|}
\hline & \multicolumn{3}{|l|}{ Treatment } \\
\hline & None & Rat lgG & $5 C 6$ \\
\hline Spleen weight (g) & $0.20 \pm 0.05$ & $0.19 \pm 0.04$ & $0.19 \pm 0.02$ \\
\hline \multicolumn{4}{|l|}{ Spleen cell populations (\%) } \\
\hline B220 $\mathrm{B}$ cells/total cells & $48.0 \pm 2.7$ & $47.7 \pm 3.3$ & $56.4 \pm 1.9^{\mathrm{b}}$ \\
\hline CD69 ${ }^{+}$B220 ${ }^{+}$B cells/total B cells & $5.3 \pm 1.9$ & $4.5 \pm 1.0$ & $2.7 \pm 0.2^{b}$ \\
\hline $\mathrm{PNA}^{+} \mathrm{B}^{2} 20^{+}$B cells/total B cells & $3.8 \pm 1.2$ & $3.9 \pm 0.5$ & $1.2 \pm 0.1^{c}$ \\
\hline CD138 ${ }^{+}$plasma cells/total cells & $2.1 \pm 0.5$ & $1.7 \pm 0.2$ & $1.1 \pm 0.2^{d}$ \\
\hline $\mathrm{CD}^{+}{ }^{+} \mathrm{T}$ cells/total cells & $17.5 \pm 0.4$ & $16.2 \pm 0.8$ & $17.3 \pm 0.7$ \\
\hline $\mathrm{CD} 9^{+} \mathrm{CD}^{+} \mathrm{T}$ cells/total $\mathrm{T}$ cells & $35.9 \pm 4.2$ & $29.9 \pm 2.5$ & $34.64 \pm 1.53$ \\
\hline CD11 b cells/total cells & $12.0 \pm 2.5$ & ND & $9.1 \pm 1.5$ \\
\hline CD11 $^{+}$cells/total cells & $6.9 \pm 1.3$ & ND & $5.8 \pm 0.6$ \\
\hline \multicolumn{4}{|l|}{ Peripheral blood (\%) } \\
\hline Neutrophils/total cells & $16.8 \pm 1.7$ & ND & $9.5 \pm 2.4$ \\
\hline Monocytes/total cells & $54.0 \pm 2.3$ & $50.5 \pm 11.9$ & $41.3 \pm 1.8^{\mathrm{b}}$ \\
\hline $\mathrm{Gr}-1^{+}$monocytes/total cells & $6.2 \pm 1.4$ & ND & $4.4 \pm 0.6$ \\
\hline Gr-1- monocytes/total cells & $47.8 \pm 1.7$ & ND & $37.0 \pm 1.8^{\mathrm{e}}$ \\
\hline
\end{tabular}

ND not defined, 5 C6 anti-mouse CD11b monoclonal antibody, KO1 FcyRIIB-deficient mouse strain

${ }^{a}$ Values are the mean \pm SEM of at least 6 female mice aged 10 months

${ }^{b}$ Differences were statistically significant versus untreated KO1 and versus normal rat lgG-treated KO1 (analysis of variance (ANOVA) $P<0.05$ )

${ }^{c}$ Differences were statistically significant versus untreated KO1 and versus normal rat lgG-treated KO1 (ANOVA $P<0.01$ )

${ }^{\mathrm{d} D i f f e r e n c e s ~ w e r e ~ s t a t i s t i c a l l y ~ s i g n i f i c a n t ~ v e r s u s ~ u n t r e a t e d ~ K O 1 ~(A N O V A ~} P<0.01$ ) and versus normal rat IgG-treated KO1 (ANOVA $P<0.05$ )

eDifference was statistically significant versus untreated KO1 (Student's $t$ test $P<0.05$ )

groups of mice were evaluated as fold change compared with the level in B6 mice tentatively designated as 1. As shown in Fig. 4, compared with untreated and normal rat IgG-treated KO1 mice, the expression levels of RANK and RANKL were significantly lower in 5C6treated $\mathrm{KO} 1$ mice. In contrast, the OPG expression level in 5C6-treated KO1 mice was significantly higher than that in untreated KO1 mice. Furthermore, when calculating the RANKL/OPG ratio, the average ratio in 5C6treated $\mathrm{KO} 1$ mice was much lower compared with those in the two control groups of mice.

Three chemokines, MCP- 1 , regulated on activation, normal $\mathrm{T}$ cell expressed and secreted (RANTES), and $\mathrm{CX}_{3} \mathrm{C}$ chemokine ligand 1 (CX3CL1), have been shown to play important roles in the pathogenesis of RA [16]; therefore we compared their expression levels in ankle joint tissue. The expression MCP-1 and RANTES, but not CX3CL1, was down-regulated in 5C6-treated mice, compared with those in the two control groups of mice (Fig. 4). Thus, it appears that MCP-1 and RANTES play pivotal roles in the inflammatory process in the arthritic joints of $\mathrm{KO} 1$ mice.

Figure 4 also compares the expression levels of TNF $\alpha$, IL-6, IL-17, and IL-10 among the three groups of mice. The levels of TNF $\alpha$ and IL- 6 were markedly downregulated in 5C6-treated mice, compared with the levels in untreated and normal rat IgG-treated KO1 mice. No significant differences were observed in IL-17 and IL-10 expression levels among the three groups of mice. These findings are consistent with our previous reports showing that both IL- 6 and TNF $\alpha$ are indispensable in the pathogenesis of arthritis in KO1 mice [11, 12].

\section{Effect of 5C6 treatment on B cell activation}

As shown in Table 2, B cells were activated and differentiated in untreated and rat IgG-treated $\mathrm{KO} 1$ mice, and these were significantly suppressed in 5C6-treated $\mathrm{KO} 1$ mice. To evaluate the effect of $5 \mathrm{C} 6$ treatment on the expression levels of $\mathrm{B}$ cell activation/differentiation-related cytokines, such as B-cell-activating factor of the tumornecrosis-factor family (BAFF), IL- $1 \beta$, B cell stimulating factor-3 (BSF-3), IL-6, and IL-10, qRT-PCR analysis was performed using mRNA extracted from the spleens of the 10-month-old mice. Data on normal B6 mice were used as a relative control. As shown in Fig. 5a, the expression levels of the former four cytokines were markedly up-regulated in untreated and rat IgG-treated mice, while the levels were significantly suppressed by $5 \mathrm{C6}$ treatment. We also compared the expression levels of IL-17, IFN- $\gamma$, and TNF $\alpha$ among the three groups of mice. The levels of IL-17 and TNF $\alpha$ were up-regulated in the control groups, and were significantly suppressed in 5C6-treated $\mathrm{KO} 1$ mice.

It has been shown that in lupus-prone mice the frequency of peripheral monocytes correlates well with the 


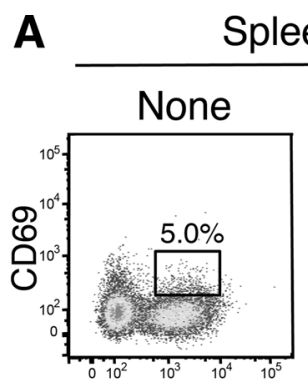

Spleen cells
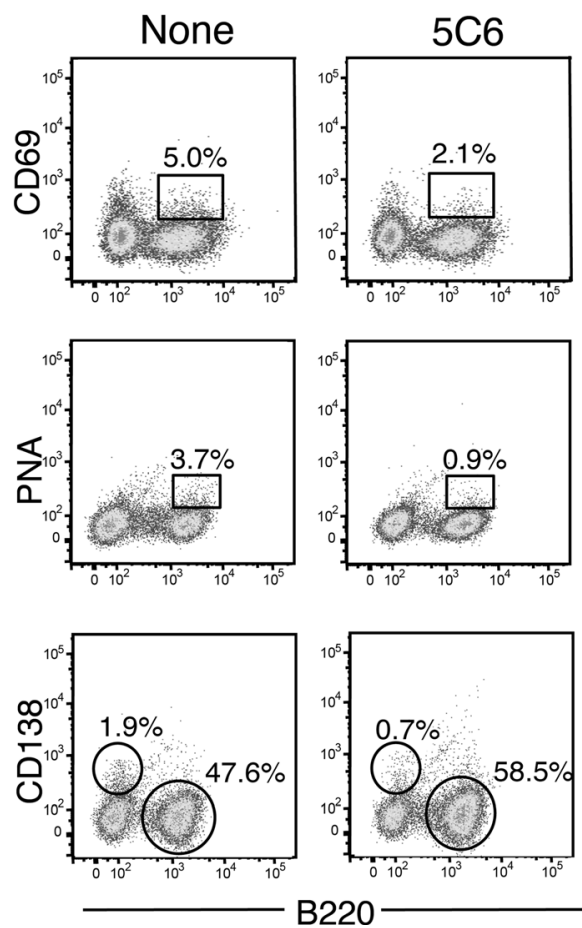

B

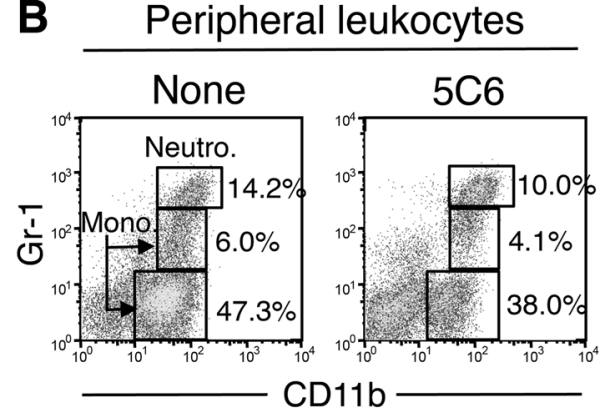

\section{CD115-gated monocytes}

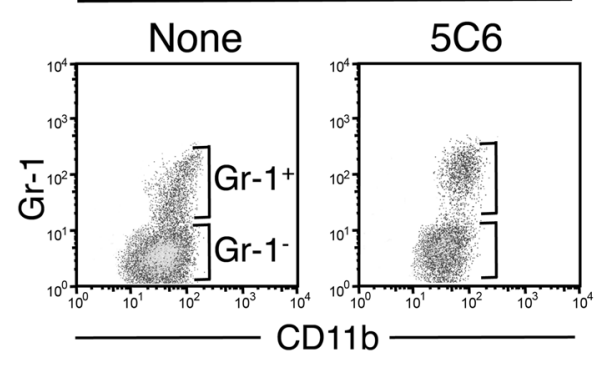

Fig. 3 Representative flow cytometric profiles of spleen cells (a), peripheral leukocytes (b), and peripheral CD115 ${ }^{+}$monocytes (c) in untreated and anti-mouse CD11b monoclonal antibody (5C6)-treated FcyRIIB-deficient mouse strain (KO1) mice at 10 months of age. a Spleen cells were stained with fluorescent-labeled CD69/B220, peanut agglutinin (PNA)/B220, and CD138/B220. The frequencies of CD69 ${ }^{+}$activated B cells and of PNA ${ }^{+}$ germinal center B cells per total B cells are shown as squares. The frequencies of CD138 ${ }^{+}$plasma cells and B220 $0^{+}$cells per total cells are shown as circles. b Peripheral leukocytes were stained with fluorescent-labeled Gr-1/CD11b. The frequencies of neutrophils, monocytes of Gr-1 ${ }^{+}$and Gr-1 ${ }^{-}$subsets per total cell are shown as squares. c Peripheral leukocytes were stained with fluorescent-labeled CD115/Gr-1/CD11b. Gr-1/CD11b expression profiles of CD115 ${ }^{+}$gated monocytes clearly show Gr-1 ${ }^{+}$and $\mathrm{Gr}-1^{-}$monocyte subsets

serum level of autoantibodies [17]. Although the reason for this correlation remains to be clarified, it is feasible to speculate that peripheral monocytes may function to induce B cell activation. To examine this possibility, B cell activation/differentiation-related cytokines were analyzed by qRT-PCR using mRNA extracted from peripheral leukocytes. As shown in Fig. 5b, the expression of BAFF, IL-1 $\beta$, BSF-3, and IL-6 was up-regulated in the two control groups of $\mathrm{KO} 1$ mice, and significantly suppressed in 5C6-treated KO1 mice. The expression of IL-17, IFN- $\gamma$, and TNF $\alpha$ was also up-regulated in the control groups of mice, and suppressed in 5C6-treated mice. The tendency found in peripheral leukocytes was almost the same as that observed in the spleen. Taken together, it is likely that the decrease in the expression of $\mathrm{B}$ cell activation/differentiation-related cytokines, IL-17, and TNF $\alpha$ in the spleen in 5C6-treated KO1 mice is due to the decreased frequency of peripheral monocytes due to 5C6 treatment as shown in Table 2 , and also due to the inhibited migration of peripheral monocytes into the spleen.

\section{Discussion}

In arthritis-prone $\mathrm{KO} 1$ mice, the $5 \mathrm{C} 6$ treatment initiated at the preclinical stage markedly suppressed the incidence and severity of arthritis from the early stage of the disease. This mAb has been shown to inhibit recruitment of peripheral $\mathrm{CD} 1 \mathrm{~b}^{+}$myelomonocytic cells into the inflammatory site with no cytotoxic effect [13]; however, in our experiment, the repetitive 5C6 treatment had a somewhat cytotoxic effect and induced decreased frequencies of myelomonocytic cells in the periphery. The 5C6-mediated disease suppression in the initiation stage of arthritis seems to be due to the decreased $\mathrm{CD}_{11} \mathrm{~b}^{+}$inflammatory cell migration into the joint tissues, since 5C6-treated mice had mild synovial lining cell proliferation but not inflammatory cell infiltration. In the later stage, KO1 mice developed the marked cartilage and bone destruction associated with severe synovitis, pannus formation, and up-regulated TRAP-positive osteoclast generation at the bone resorption lacuna. These destructive features were seldom observed in 5C6-treated KO1 mice due to the lack of osteoclast 


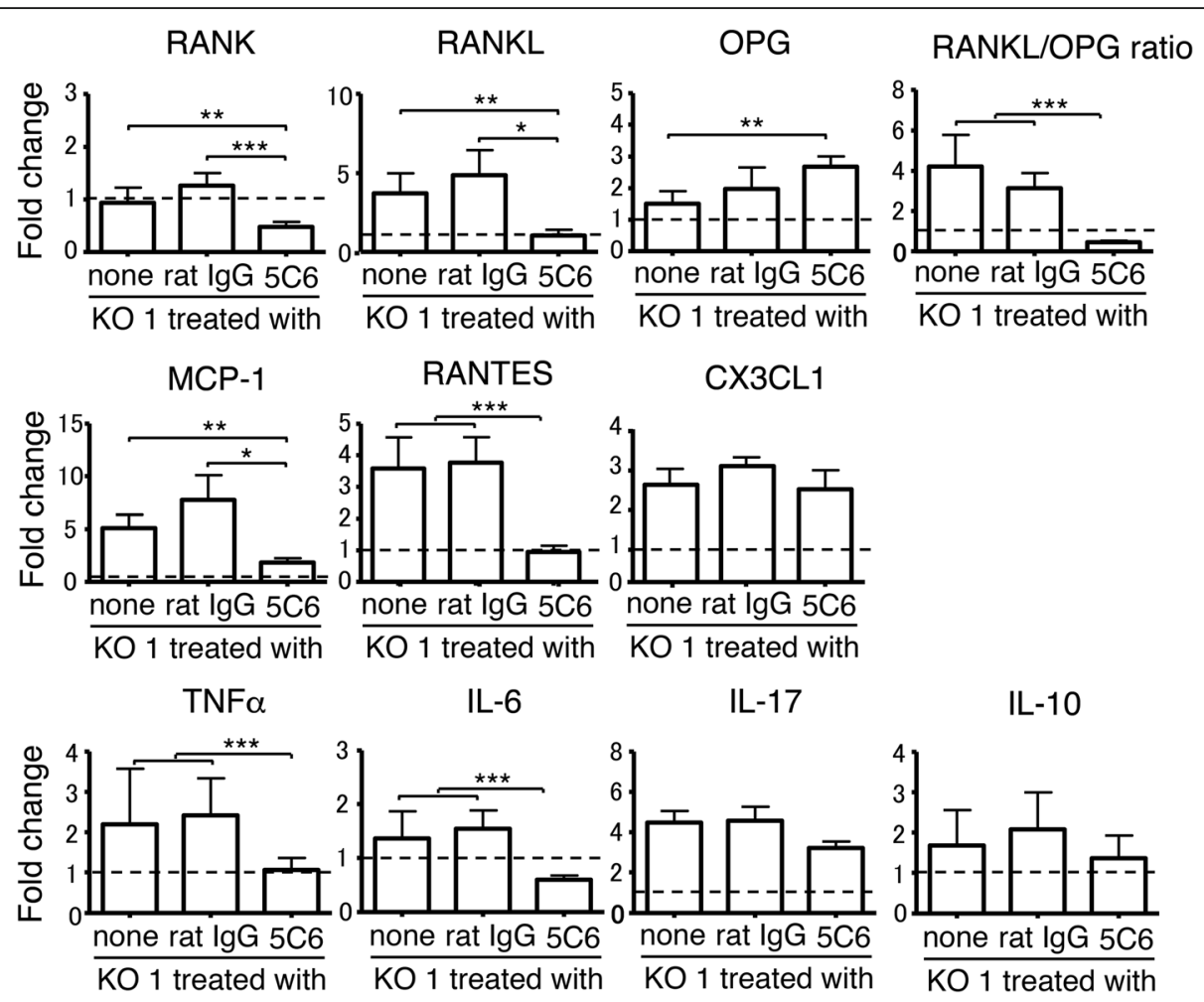

Fig. 4 Comparisons of mRNA expression levels of receptor activator of NF-KB (RANK), RANK ligand (RANKL), osteoprotogerin (OPG), RANKL/OPG ratio, monocyte chemotactic protein-1 (MCP-1), regulated on activation, normal T cell expressed and secreted (RANTES), CX3CL1, TNFa, IL-6, IL-17, and IL-10 in the ankle joints analyzed by qRT-PCR among untreated, normal rat IgG-treated, and monoclonal antibody (5C6)-treated FcyRIIB-deficient mouse strain (KO1) mice at 10 months of age. Values in each of the three groups of mice were evaluated as fold change compared with the expression levels in B6 mice (broken line). Data are shown as mean + SEM of six mice from each group. Statistical significance is shown (analysis of variance ${ }^{*} P<0.05$, $\left.{ }^{* *} P<0.01,{ }^{* * *} P<0.001\right)$

precursor monocyte recruitment into the joint tissues. The disease suppression was associated with downregulation of serum RF and of IgG autoantibodies against CCP and dsDNA. These findings clearly demonstrate that, in addition to the effect on the decreased migrations and frequencies of peripheral CD11 $\mathrm{b}^{+}$myelomonocytic cells including inflammatory cells and osteoclast precursors, $5 \mathrm{C} 6 \mathrm{mAb}$ also exerts an inhibitory effect on B cell activation/differentiation.

RANK and RANKL expression was suppressed in the joint tissue in 5C6-treated KO1 mice, while OPG was up-regulated. The decrease in RANK expression is likely due to the decreased number of RANK-expressing osteoclast precursors. The decreased RANKL expression in 5C6-treated $\mathrm{KO} 1$ mice is thought to be due to decreased IL- 6 expression, as IL- 6 signals have been shown to directly induce RANKL expression in fibroblast-like synoviocytes from patients with RA [18]. As OPG is a decoy receptor for RANKL and suppresses RANKLmediated osteoclastogenesis [8], the increased OPG expression and the lower RANKL/OPG ratio are consistent with the suppression of osteoclastogenesis in the joint tissues of 5C6-treated $\mathrm{KO} 1$ mice. OPG is produced by a variety of cells including osteoblasts, B cells, dendritic cells, and vascular endothelial cells $[19,20]$, and the marked decrease in OPG expression in arthritic joints has been suggested as a result of the exhaustion of OPG production due to chronic stimulation by TNF $\alpha$ over the extended period [20]. Consistently, synovial OPG expression has been shown to be increased in patients with RA treated with anti-TNF $\alpha$ [21] and in TNF $\alpha$-deficient KO1 mice [11]. Thus, the increased level of OPG in 5C6-treated mice may be due to lower expression of TNF $\alpha$.

RA is an autoantibody-mediated autoimmune disease, in which IgG immune complexes (ICs) trigger inflammatory myelomonocytic cell activation via IC-binding to the FcR $\gamma$ chains of stimulating IgG Fc receptors [22, 23]. These activated inflammatory cells produce several inflammatory cytokines including chemokines, which induce additional inflammatory cell infiltration in the joint tissues. Our arthritis-prone $\mathrm{KO} 1$ mice lack the expression of inhibitory FcyRIIB molecules [10], which are usually expressed on B cells and myelomonocytic cells. The FcyRIIB molecules inhibit the activation signals mediated by the B cell antigen receptors on B cells and by the FcR $\gamma$ chains on myelomonocytic cells [22, 23]. The lack of 

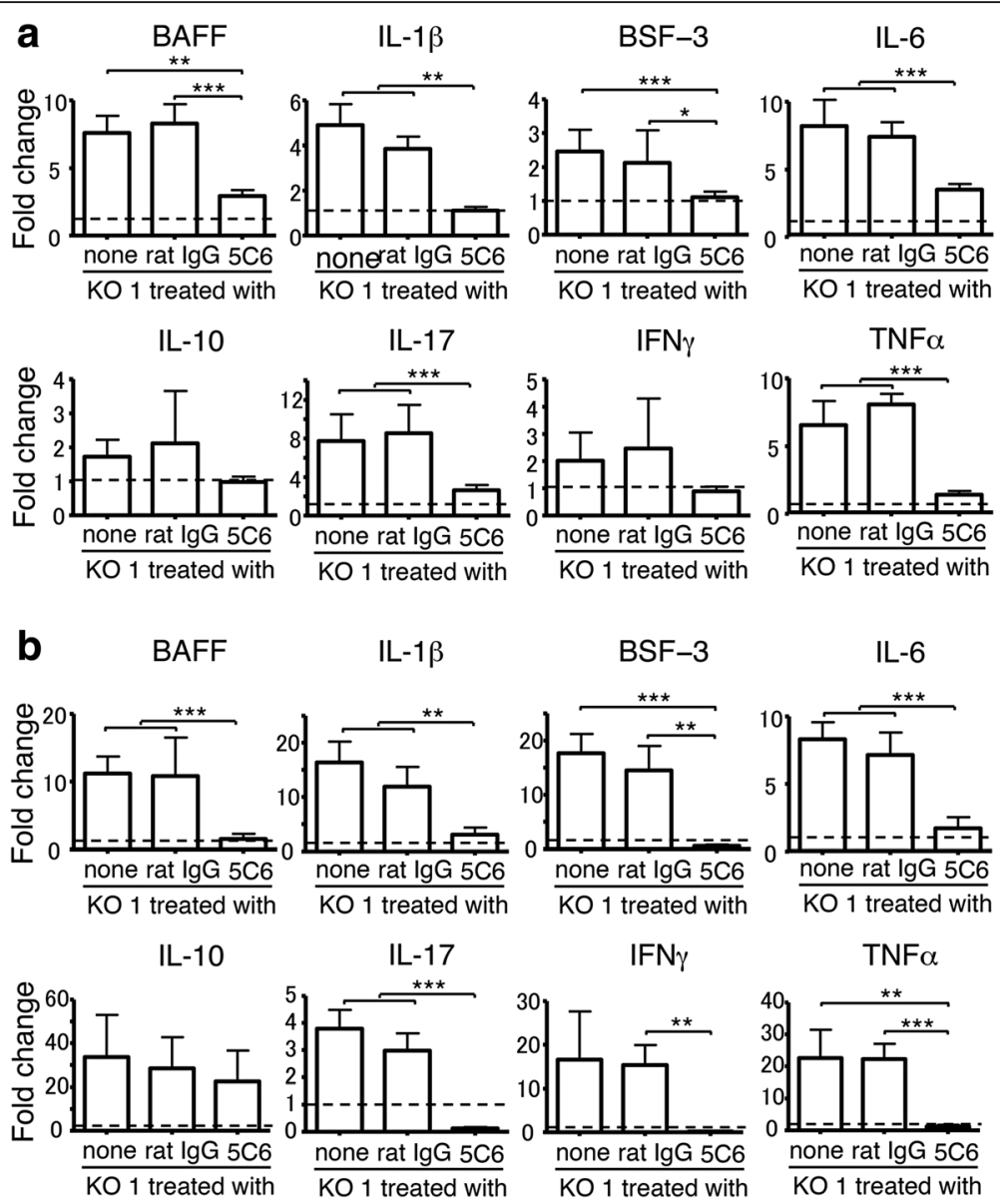

Fig. 5 Comparisons of mRNA expression levels of B-cell-activating factor of the tumor-necrosis-factor family (BAFF), IL-1 $\beta, B$ cell stimulating factor-3 (BSF-3), IL-6, IL-10, IL-17, interferon- $\gamma($ IFN- - ), and TNFa in spleen (a) and peripheral leukocytes (b) analyzed by qRT-PCR among untreated, normal rat IgG-treated, and monoclonal antibody (5C6)-treated FcyRIIIB-deficient mouse strain (KO1) mice at 10 months of age. Values of each of the three groups of mice were evaluated as fold change compared with the expression levels in B6 mice (broken line). Data are shown as mean + SEM of six mice from each group. Statistical significance is shown (analysis of variance ${ }^{*} P<0.05,{ }^{* *} P<0.01,{ }^{* * *} P<0.001$ )

FcyRIIB expression on B cells induces the breakdown of self-tolerance and the production of IgG autoantibodies [24]. The resultant IgG ICs formed in the joint tissues stimulate myelomonocytic cells via the FcR $\gamma$ chains, and this process is augmented in $\mathrm{KO} 1$ mice. In 5C6-treated $\mathrm{KO} 1$ mice, the expression levels of chemokines such as MCP-1 and RANTES were significantly down-regulated, indicating that these cytokines play important roles in the pathogenesis of arthritis in $\mathrm{KO} 1$ mice. Intriguingly, it has been shown that once $\mathrm{RANK}^{+}$osteoclast precursor monocytes are activated via membrane-bound RANKL, it leads to increased expression of MCP-1 and RANTES, and these chemokines act as chemical signals, attracting other monocytes to the site of RANKL expression. These chemokines promote fusion of monocytes with RANKL-stimulated osteoclast precursors, resulting in the generation of multinucleated mature osteoclasts [25].

Peripheral monocytes are composed of heterogeneous populations and could mainly be subdivided into two phenotypically and functionally distinct subsets [4, 26]. In mice, monocytes are clearly divided into two populations, namely $\mathrm{Gr}-1^{+}$and $\mathrm{Gr}-1^{-}$subsets. The former is CX3CR $1{ }^{\text {low } C C R} 2^{+}$and is called an "inflammatory" subset that is actively recruited to inflamed tissues via MCP-1, the ligand for CCR2, whereas the latter is CXCR $3{ }^{\text {high }}$ CCR 2 " and called a "resident" subset that is recruited to non-inflamed tissues. Intriguingly, in lupusprone mice the $\mathrm{Gr}-1^{-}$, but not the $\mathrm{Gr}-1^{+}$subset is selectively expanded and expresses the stimulatory FcyRIV, suggesting that the $\mathrm{Gr}-1^{-}$subset may be in a more active stage than the $\mathrm{Gr}-1^{+}$subset, and may contribute to kidney damage [27]. Compared with normal B6 mice with monocyte frequencies below 10\% [17], our arthritis-prone KO1 mice develop marked monocytosis with frequencies above $50 \%$, mainly composed of the $\mathrm{Gr}-1^{-}$subset. The $5 \mathrm{C} 6$ treatment especially suppressed the frequency of $\mathrm{Gr}-1^{-}$ monocytes, suggesting the important role of the $\mathrm{Gr}-1^{-}$ subset in osteoclastogenesis. Thus, the originally classified 
Gr-1- "resident" subset may contain functionally different cell types, one responsible for lupus nephritis and another for osteoclastogenesis in the joints. However, there are conflicting results reported on the cell types that can give rise to osteoclasts. Yao et al. [28] report that the $\mathrm{Gr}-\mathrm{1}^{-}$subset is the major precursor of osteoclasts. In contrast, Seeling et al. [29] showed that the $\mathrm{Gr}-1^{+}$inflammatory monocyte subset represents the major precursor of osteoclasts, and that these $\mathrm{Gr}-\mathrm{1}^{+}$monocytes differentiated into mature osteoclasts paralleled by up-regulation of FcyRIV expression upon in vitro activation via RANKL. They also showed that the cross-linking of activating FcyRIV by ICs was critical for osteoclast development. Given the important role of FcyRIV in IC-mediated stimulation, Gr-1 $1^{-}$ $\mathrm{Fc}_{\mathrm{R}} \mathrm{RIV}^{+}$monocytes may contribute to the initial step of inflammatory responses, including MCP-1 expression, in the arthritic lesion, and then $\mathrm{Gr}-1^{+} \mathrm{CCR} 2^{+}$monocytes are attracted by MCP-1, resulting in the fusion with activated Gr-1 ${ }^{-} \mathrm{Fc}$ RIV ${ }^{+}$monocytes to generate multinucleated mature osteoclasts, as suggested [25].

Genetic studies using lupus-prone mice showed that the frequency of peripheral monocytes correlates well with the serum level of autoantibodies [17]. In the present study, 5C6 treatment down-regulated the frequency of the Gr-1- monocyte subset, along with decreased expression of BAFF, IL- $1 \beta$, BSF-3, and IL- 6 in the periphery, suggesting that $\mathrm{Gr}-1^{-}$monocytes are the major producer of these cytokines in $\mathrm{KO} 1$ mice. BAFF enhances B cell survival and immune responses [30]. Although the important roles of IL-1 $\beta$ in innate immune responses and T cell differentiation are known [31], IL-1 $\beta$ is also well-recognized as an essential cytokine for antibody production and B cell proliferation [32, 33]. BSF-3, also reported as cardiotrophin-like cytokine, belongs to the IL-6 family and strongly stimulates antibody production by B cells [34, 35]. These B cell activation/differentiation-related cytokines may play a pivotal role in B cell activation and consequently in the pathogenic autoantibody production in KO1 mice. Taken together, the blocking of monocyte function may be an additional possible therapeutic strategy for rheumatoid arthritis.

\section{Conclusions}

Our originally established FcyRIIB-deficient mouse strain (designated KO1) develops severe arthritis resembling human RA. Taking advantage of treatment of KO1 mice with the $\mathrm{mAb} 5 \mathrm{C} 6$, we showed that $5 \mathrm{C} 6$ treatment results in not only amelioration of arthritis but also decrease in autoantibody production. The amelioration of arthritis was thought to be due to the decreased number and recruitment into joint tissues of peripheral monocytes, including inflammatory cells and osteoclast precursors. The down-regulated autoantibody production was thought to be due to the decreased frequencies of monocytes, which have the potential to produce $\mathrm{B}$ cell activating cytokines. The present findings indicate that the blocking of monocyte function could be an additional possible therapeutic strategy for rheumatoid arthritis.

\section{Abbreviations \\ 5C6: Anti-mouse CD11b monoclonal antibody; ANOVA: Analysis of variance; B6: C57BL/6; BAFF: B-cell-activating factor of the tumor-necrosis-factor family; BSF-3: B cell stimulating factor-3; CCP: Cyclic citrullinated peptide; CX3CL1: CX ${ }_{3} C$ chemokine ligand 1; FcyRIIB: IgG Fc receptor IIB; ELISA: Enzyme-linked immunosorbent assay; ICs: Immune complexes; IFN- $\gamma$ : Interferon- $\gamma$; IL: Interleukin; KO1: FcyRIIB-deficient mouse strain; mAb: monoclonal antibody; MCP-1: Monocyte chemotactic protein-1; OPG: Osteoprotegerin; PNA: Peanut agglutinin; RA: Rheumatoid arthritis; RANK: Receptor activator of NF-kB; RANKL: RANK ligand; RANTES: Regulated on activation, normal T cell expressed and secreted; RF: Rheumatoid factor; Th17: T helper 17; TNFa: Tumor necrosis factor a; TRAP: Tartrate-resistant acid phosphatase}

\section{Acknowledgements \\ Not applicable.}

\section{Funding}

This work was supported in part by a Grant-in-Aid (15 K08432) from the Ministry of Education, Science, Technology, Sports and Culture of Japan; and a grant for Research on Intractable Diseases from the Ministry of Health, Labor and Welfare of Japan.

Availability of data and materials

Not applicable.

\section{Authors' contributions}

$\mathrm{MO}$ conducted the study procedures and reviewed and approved the manuscript; QL helped to conduct the study and reviewed and approved the manuscript; $\mathrm{HO}$ helped to conduct the study and reviewed and approved the manuscript; KT helped to conduct the study and reviewed and approved the manuscript; HA helped to conduct the study and reviewed and approved the manuscript; HY provided $5 \mathrm{C} 6 \mathrm{mAb}$ and reviewed and approved the manuscript; HN helped to conduct the study and reviewed and approved the manuscript; SH designed the study, conducted the analyses, and drafted, reviewed, and approved the manuscript.

\section{Ethics approval}

All animal experiments were approved by the Institutional Animal Care and Use Committee at the Toin University of Yokohama under the protocol reference number D29-16.

Consent for publication

Not applicable.

Competing interests

The authors declare that we have no competing interests.

\section{Publisher's Note}

Springer Nature remains neutral with regard to jurisdictional claims in published maps and institutional affiliations.

\section{Author details}

${ }^{1}$ Department of Biomedical Engineering, Toin University of Yokohama, 1614 Kurogane-cho, Aoba-ku, Yokohama 225-8502, Japan. ${ }^{2}$ Health and Life Science, Musashigaoka Junior College, Saitama 355-0154, Japan. ${ }^{3}$ Faculty of Health and Welfare, Kanagawa University of Human Services, Yokosuka 238-8522, Japan. ${ }^{4}$ Department of Rheumatology and Internal Medicine, Juntendo University School of Medicine, Tokyo 113-8421, Japan.

${ }^{5}$ Department of Immunology, Juntendo University School of Medicine, Tokyo 113-8421, Japan. 
Received: 5 September 2017 Accepted: 19 January 2018 Published online: 08 February 2018

\section{References}

1. Connor JR, Dodds RA, James IE, Gowen M. Human osteoclast and giant cell differentiation: the apparent switch from nonspecific esterase to tartrate resistant acid phosphatase activity coincides with the in situ expression of osteopontin mRNA. J Histochem Cytochem. 1995;43:1193-201.

2. Dodds RA, Connor JR, Drake FH, Gowen M. Expression of cathepsin K messenger RNA in giant cells and their precursors in human osteoarthritic synovial tissues. Arthritis Rheum. 1999;42:1588-93.

3. Boyle W, Simonet WS, Lacey DL. Osteoclast differentiation and activation. Nature. 2003;423:337-42.

4. Gordon S, Taylor PR. Monocyte and macrophage heterogeneity. Nat Rev Immunol. 2005;5:953-64.

5. Takayanagi H. Osteoimmunology and the effect of the immune system on bone. Nat Rev Rheumatol. 2009;5:667-76.

6. Walsh MC, Kim N, Kadono Y, Rho J, Lee SY, Lorenzo J, et al. Osteoimmunology: interplay between the immune system and bone metabolism. Annu Rev Immunol. 2006;24:33-63.

7. Sato K, Suematsu A, Okamoto K, Yamaguchi A, Morishita Y, Kadono Y, et al. Th17 functions as an osteoclastogenic helper T cell subset that links $T$ cell activation and bone destruction. J Exp Med. 2006;203:2673-82.

8. Simonet WS, Lacey DL, Dunstan CR, Kelley M, Chang MS, Luthy R, et al. Osteoprotegerin: a novel secreted protein involved in the regulation of bone density. Cell. 1997:89:309-19.

9. Mclnnes JB, Schett G. Cytokines in the pathogeneses of rheumatoid arthritis. Nat Rev Immunol. 2007;7:429-42.

10. Sato-Hayashizaki A, Ohtsuji M, Lin Q, Hou R, Ohtsuji N, Nishikawa K, et al. Presumptive role of 129 strain-derived Sle16 locus in rheumatoid arthritis in a new mouse model with Fcy receptor Type Ilb-deficient C57BL/6 genetic background. Arthr Rheum. 2011;63:2930-8.

11. Okazaki H, Lin Q, Nishikawa K, Ohtsuji N, Tsurui H, Ohtsuji M, et al. TNFa but not IL-17 is critical in the pathogenesis of rheumatoid arthritis spontaneously occurring in a unique FcyRllB-deficient mouse model. Mod Rheumatol. 2014;24:931-8.

12. Ohtsuji M, Lin Q, Nishikawa K, Ohtsuji N, Okazaki H, Tsurui H, et al. IL-6 signal blockade ameliorates the enhanced osteoclastogenesis and the associated joint destruction in a novel FcyRIIB-deficient rheumatoid arthritis mouse model. Mod Rheumatol. 2015;25:270-7.

13. Rosen H, Gordon S. Monoclonal antibody to the murine type 3 complement receptor inhibits adhesion of myelomonocytic cells in vitro and inflammatory cell recruitment in vivo. J Exp Med. 1987;166:1685-701.

14. Wipke BT, Allen PM. Essential role of neutrophils in the initiation and progression of a murine model of rheumatoid arthritis. J Immunol. 2001;167:1601-8.

15. Abe Y, Ohtsuji M, Ohtsuji N, Lin Q, Tsurui H, Nakae S, et al. Ankylosing enthesitis associated with up-regulated IFN- $\gamma$ and IL-17 production in (BXSB X NZB) F1 male mice: a new mouse model. Mod Rheumatol. 2009;19:316-22.

16. Iwamoto T, Okamoto H, Toyama Y, Momohara S. Molecular aspects of rheumatoid arthritis: chemokines in the joints of patients. FEBS J. 2008;275:4448-55.

17. Kikuchi S, Santiago-Raber M-L, Amano H, Amano E, Fossati-Jimack L, Moll T, et al. Contribution of NZB autoimmunity 2 to Y-linked autoimmune acceleration-induced monocytosis in association with murine systemic lupus. J Immunol. 2006;176:3240-7.

18. Hashizume M, Hayakawa N, Mihara M. IL-6 trans-signalling directly induces RANKL on fibroblast-like synovial cells and is involved in RANKL induction by TNF-a and IL-17. Rheumatology. 2008:47:1635-40.

19. Yun TJ, Chaudhary PM, Shu GL, Frazer JK, Ewing MK, Schwartz SM, et al. OPG/FDCR-1, a TNF receptor family member, is expressed in lymphoid cells and is up-regulated by ligating CD40. J Immunol. 1998;161:6113-21.

20. Zannettio ACW, Holding CA, Diamond P, Atkins GJ, Kostakis P, Farrugia A, et al. Osteoprotegerin (OPG) is localized to the Weibel-Palade bodies of human vascular endothelial cells and is physically associated with von Willebrand factor. J Cell Physiol. 2005;204:714-23.

21. Catrina Al, Klint E, Ernestam S, Catrina S-B, Makrygiannakis D, Botusan IR, et al. Anti-tumor necrosis factor therapy increases synovial osteoprotegerin expression in rheumatoid arthritis. Arthritis Rheum. 2006;54:76-81.

22. Takai T. Roles of Fc receptors in autoimmunity. Nat Rev Immunol. 2002;2:580-92.
23. Ravetch JV, Bolland S. IgG Fc receptors. Annu Rev Immunol. 2001;19:275-90.

24. Bolland S, Ravetch JV. Spontaneous autoimmune disease in FcyRIII-deficient mice results from strain-specific epistasis. Immunity. 2000;13:277-85.

25. Kim MS, Day CJ, Morrison NA. MCP-1 is induced by receptor activator of nuclear factor-kB ligand, promotes human osteoclast fusion, and rescues granulocyte macrophage colony-stimulating factor suppression of osteoclast formation. J Biol Chem. 2005;280:16163-9.

26. Geissmann F, Jung S, Littman DR. Blood monocytes consist of two principal subsets with distinct migratory properties. Immunity. 2003;19:71-82.

27. Santiago-Raber M-L, Amano H, Amano E, Baudino L, Otani M, Lin Q, et al. FcyR-dependent expansion of a hyperactive monocyte subset in lupus-prone mice. Arthritis Rheum. 2009;60:2408-17.

28. Yao Z, Li P, Zhang Q, Schwarz EM, Keng P, Arbini A, et al. Tumor necrosis factor-a increases circulating osteoclast precursor numbers by promoting their proliferation and differentiation in the bone marrow through up-regulation of c-Fms expression. J Biol Chem. 2006;281:11846-55.

29. Seeling M, Hillenhoff U, David JP, Schett G, Tuckermann J, Lux A, et al. Inflammatory monocytes and Fcy receptor IV on osteoclasts are critical for bone destruction during inflammatory arthritis in mice. Proc Nalt Acad Sci USA. 2013;110:10729-34.

30. Mackey F, Browning JL. BAFF: a fundamental survival factor for B cells. Nat Rev Immunol. 2002:2:465-75.

31. Sims JE, Smith DE. The IL-1 family: regulators of immunity. Nat Rev Immunol. 2010;10:89-102.

32. Nakae $S$, Asano M, Horai $R$, Iwakura $Y$. Interleukin-1 $\beta$, but not interleukin-1a, is required for T-cell-dependent antibody production. Immunology. 2001;104:402-9.

33. Maliszewski CR, Sato TA, Vanden Bos T, Waugh S, Dower SK, Slack J, et al. Cytokine receptors and $B$ cell functions. I. Recombinant soluble receptors specifically inhibit IL-1- and IL-4-induced B cell activities in vitro. J Immunol. 1990;144:3028-33.

34. Senaldi G, Varnum BC, Sarmiento U, Starnes C, Lile J, Scully S, et al. Novel neurotrophin-1/B cell-stimulation factor-3: a cytokine of the IL-6 family. Proc Nalt Acad Sci USA. 1999;96:11458-63.

35. Senaldi G, Stolina M, Guo J, Faggioni R, McCabe S, Kaufman SA, et al. Regulatory effects of novel neurotrophin-1/b cell-stimulating factor-3 (cardiotrophin-like cytokine) on B cell function. J Immunol. 2002;168:5690-8.

\section{Submit your next manuscript to BioMed Central and we will help you at every step:}

- We accept pre-submission inquiries

- Our selector tool helps you to find the most relevant journal

- We provide round the clock customer support

- Convenient online submission

- Thorough peer review

- Inclusion in PubMed and all major indexing services

- Maximum visibility for your research

Submit your manuscript at www.biomedcentral.com/submit
C Biomed Central 